\title{
Removing old chondrocytes to combat disease
}

Senescent cells accumulate in a variety of tissues as the body ages, releasing a selection of pro-inflammatory factors that can contribute to tissue damage. In patients with osteoarthritis (OA), senescent cells exist in cartilage tissue, but their role in disease progression is poorly understood. Now, new research reveals a potential pathogenic role for senescent cells in OA that can be countered by depleting these cells from affected joints.

The researchers behind this new study utilized the p16-3MR transgenic mouse, which enables the luciferase-based tracking and ganciclovir-induced selective killing of cells expressing Cdkn2a (also known as P16ink4a), a gene upregulated in senescent cells. By monitoring Cdkn2aexpressing cells in mice that have undergone surgery on their anterior cruciate ligament (ACL), a model of post-traumatic OA, the research team observed that cells in the joint tissues became senescent following injury and secreted pro-inflammatory factors. Removing these senescent cells reduced pain and the accrual of cartilage erosion, as well as increasing cartilage development.

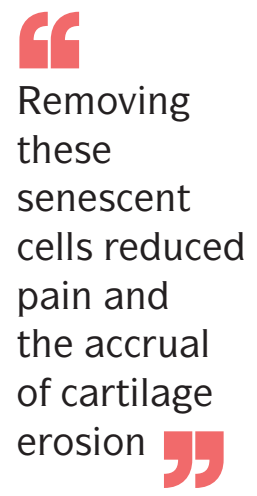

Following ACL surgery in young (10 week-old) p16-3MR mice, senescent cells accumulated in the superficial layer of cartilage and in the synovium and secreted a range of pro-inflammatory cytokines and matrix-degrading enzymes. Selective depletion of the Cdkn2a-expressing senescent cells by ganciclovir not only inhibited articular cartilage erosion and reduced the expression of pro-inflammatory factors, but also increased the production of type II collagen and aggrecan, suggestive of cartilage repair. However, depletion of senescent cells did not rescue the damage accrued in the subchondral bone.

By contrast, aged (19 month-old) p16-3MR mice exhibited more severe OA than their young counterparts following ACL surgery. Senescent cells were distributed throughout the cartilage in aged mice, rather than primarily in the superficial zone, and depletion of these cells reduced disease activity without creating the pro-regenerative environment observed in young mice.

The researchers replicated the ganciclovir-induced depletion of senescent cells in young p16-3MR and C57BL mice following ACL surgery using the senolytic agent
UBX0101, which selectively kills senescent cells. Removal of senescent cells by UBX0101 mirrored the effects observed upon depletion of senescent cells in young p16-3MR mice by ganciclovir; articular cartilage erosion, pain and levels of pro-inflammatory factors were reduced and cartilage production increased, but subchondral bone was unaffected.

"We are now looking more into the impact of these cells in older animals and the impact of trauma and age. Also, we are looking at how these cells [recruit] immune cells to the area of damage and the role of these immune cells in disease progression," states Jennifer Elisseeff, corresponding author on the study. Looking to the future, the researchers also hope to determine the optimal drug and dosage for selectively clearing senescent cells from the cartilage of patients with $\mathrm{OA}$.

Joanna Collison

ORIGINAL ARTICLE Jeon, O. H. et al. Local clearance of senescent cells attenuates the development of post-traumatic osteoarthritis and creates a pro-regenerative environment. Nat. Med. http://dx.doi.org/10.1038/nm.4324 (2017) FURTHER READING Loeser, R. F. et al. Ageing and the pathogenesis of osteoarthritis. Nat. Rev. Rheumatol. 12, 412-420 (2016) 